\section{JCL}

Journal of the College of Languages

Open Free Access, Peer Reviewed Research Journal

http://icolang.uobaghdad.edu.iq
P-ISSN: 2074-9279

E-ISSN: $2520-3517$ 2019, No.(39)

Pg.129-144

\title{
Las Dificultades en la Enseñanzayel Aprendizaje de Español como Lengua Extranjera (ELE)en Particular ylas Lenguas Extranjeras en General: El Docente no Nativo
}

\section{Difficulties and Problems in Teaching and Learning Spanish in Particular and Foreign Languages in General: The Teaching Staff}

Inst. Awad Hussein Nada, Ph.D. email: awadhussein2002@yahoo.es Ministry of Scientific Research and Higher Education, Directorate of Scholarships and Cultural Relations.

\section{(Received on 23/5/2018 : Accepted on 20/6/2018)}

\begin{abstract}
This paper aims at providing the teaching staff members with the necessary skills so as to become capable of tackling various situations, and treating daily problems that face students learning Spanish as a Second Language. This is made as an attempt to make teachers of foreign languages in general acquainted with modern trends of teaching with less complicated methods, specifically in teaching e earlier stages of foreign languages.
\end{abstract}

Key words: Teaching, Foreign language, Spanish, Teaching Staff, Methods of Teaching. 


\title{
Las Dificultades en la Enseñanzayel Aprendizaje de Español como Lengua Extranjera (ELE)en Particular ylas Lenguas Extranjeras en General: El Docente no Nativo
}

\author{
Por Inst. Awad Hussein Nada (Ph.D.) \\ Ministerio de Educación Superior e \\ Investigación Científica- Dirección de \\ Becas y Relaciones Culturales
}

\begin{abstract}
o:
En el presente trabajo pretendemos dotar al docente no nativo de Lenguas extranjeras, con algunos de los métodos necesarios para poder actuar, junto con sus alumnos, en una variedad de situaciones y enfrentarse a los problemas cotidianos, de manera que desarrolle las destrezas comunicativas del alumnado y establecer una comunicación exitosa,. Así pues, nuestro trabajo será un intento de posibilitar al profesorado a tomar en consideración, reflexionar y familiarizase conlas tendencias modernas de enseñanza con métodos menos complicados, sobre todo, en las primeras fases del aprendizaje.
\end{abstract}

Palabras claves: enseñanza, lengua extranjera, docente no nativo, actuación, metodología.

\section{Prefacio.}

No cabe duda que la enseñanza de lenguas extranjeras siempre ha desempeñado un papel muy importante en el mundo, porque la necesidad de comunicarsee intercambiarlasideas y los conocimientosno se pueden realizar sin conocer las lenguas. En nuestra épocael númerode los estudiantes que quieren aprender el español como lengua extranjera ha aumentado. Esteascenso de aprendizaje requiere cambios en los métodos de enseñanza y en los programas educativos, porque la calidad del aprendizaje, ha sido uno de los objetivos primordiales de los estudiantes. Por ello,ha sido necesario 
fomentar a los docentes con los métodos actualizados. En general podemos decir queenseñar una lengua no basta con ser un profesor nativo o dominar bien la lengua. Es preciso, también ser capaz de decidir cuándo es más conveniente trabajar en grupos, en parejas o individualmente, como mantener losintereses y la motivación de sus alumnos, además de todo esto, tendría que poseer buenos conocimientos en todas las disciplinas de la lingüística aplicada, lingüística contrastiva, gramática normativa y en didáctica de lenguas y por si fuera poco un buen manejo de la últimas tecnologías y sus aplicaciones en la enseñanza.Los profesores se encuentran con diversas situaciones de aprendizaje, difíciles de entender tanto a nivel del estudiante como al nivel de la metodología utilizada y para poder solucionarlas esos entresijos, necesitan una formación adecuada. Además, resulta conveniente saber elaborar unidades didácticas y materiales para hacer su tarea según las normativasvigentes (por ejemplo El Plan Curricular del Instituto de Cervantes). Y no hay que olvidar que enseñar y aprender una lengua extranjera dentro del contexto de la cultura es más importante cuando uno está involucrado en la lengua meta fuera de su entorno real. En fin los problemas de enseñanza no se pueden restringir en un número determinable de métodos y teorías, por eso y dada la amplitud del tema en nuestro estudio intentaremos plantear un problema,que creemos que es más básico y que enfrenta al docente durante la enseñanza de español como segunda lengua o lengua extranjera ${ }^{1}$ (a partir de ahora LE)en Irak .El problema es la actuación del profesor en el aula, tomando en consideración de la existencia de muchos factoresque entran en el juego tales como: la lengua materna de los alumnos y el monolingüismo, la reacción contra el resto de las lenguas extranjeras (todo el mundo aprende inglés), y la necesidad de crear un entorno parecido al real que posibilita al alumnado a integrar la gramática con la cultura de la lengua meta en el aula ya que los estudiantes salen de sus clases y hablan en árabe y se encuentran con una cultura puramente árabe.

\section{1- La actuación del docente en el aula}

Todos estamos convencidos de que la actuación positiva del docente aumenta la motivación de sus estudiantes. Las investigaciones que establecen la relación entre la actitud positiva y el éxito en el aprendizaje de 
una segunda lengua dan soporte a este trabajo, aunque es necesario comprender que entran en el juego muchos elementos tal como la edad ${ }^{2}$, los factores psico-sociales, etc. Para empezar tenemos primero echar un vistazo somero sobre la clasificación de la naturaleza de los profesores. En el proceso del aprendizaje, la actuación de los profesores constituye uno de los ejes imprescindibles que influyen en la participación y en el rendimiento de los estudiantes. Según la forma de actuar del profesorado en el aula de lengua extranjera, presentamos unos cuantos tipos de profesores que han sido clasificados por los educadores tales como los dos alemanes Georg Kerschensteiner (1949:89) y Lorenzo Luzuriaga (1957: 145), Rubio Gel y Álvarez Irarreta (2010:42).

- Profesor/a solicito: que se refiere al tipo de los profesores que siempre deciden desarrollar las actividades sin dejar a los alumnos una libertad para elegir ni expresar u opinar. El citado comportamiento puede sacar a la luz unos alumnos tímidos y guiados por las decisiones de su profesor. Que a su vez genera un tipo de actividades rutinarias

- Profesor/a dominador "que lo sabe todo" que es prepotente que cree tener la respuesta para todo, queriendo llevar siempre la razón, su falta de modestia puede llevar a reprimir la participación de los alumnos.

- Profesor/a indolente que es contrario de los dos anteriores. Es tipo de profesores deja demasiada libertad y apenas aparece en el proceso del aprendizaje. Pero el indolente crea una situación caótica en lo que solo los estudiantes más cualificados puedan llegar a adquirir los conocimientos y las técnicas de aprendizaje.

- Profesor/a ponderado que es un tipo mixto entre los tres anteriores. El ponderado participa más, prepone actividades interesantes y siempre procura mantener una buena imagen.

- Profesor/a autoritario cuya tarea se centra en la disciplina e impide cualquier intervención o critica del alumnado

- Profesor/a nato que intenta satisfacer sus necesidades y redibuja las actividades según las características de cada alumno, e intenta hacer unos cambios cuantitativos y cualitativos en el comportamiento del alumnado.

- Profesor/a liberal que ve los alumnos como segundo sujeto en el proceso, partidario de la participación y la comunicación de los estudiantes entre sí en el aula, y deja mucho tiempo expresar sus dudas y sus opiniones. 
- Profesor/a laborioso a este tipo le interesa la planificación y la organización en la clase. También da gran importancia a las mínimas detalles de cada unidad didáctica, trata de utilizar nuevos métodos y estrategias aptos para motivar a los alumnos. Es partidario de la participación de los alumnos en el aula.

- Profesor/a demasiado ambicioso que a veces tiene intereses muy por encima de sus alumnos, lo que produce desmotivación y desánimo.

- El cordial que se caracteriza por su buena relación con el alumno y su entusiasmo por la enseñanza, y procura mucho en el tema de la intervención del alumnado en el proceso de aprendizaje.

Después de esta exposición presentada por los autores mencionados antes, podemos decir que la actitud del profesor del español como lengua extranjera tiene que ser una combinación de las características mencionadas pero hay que elegir solamente las positivas y sobre todo las que se centran el progreso de los alumnos y en el desarrollo de sus capacidades comunicativas, aunque esto no es una tarea tan fácil, porque la labor implica dibujar unas estrategias de aprendizaje que abarcan varios aspectos. La experiencia del docente, seguramente, contribuye a mejorar esta situación si existe una preparación y una actualización.

La cuestión del docente nos lleva a otro tema que es el de los profesores nativos / no nativos. En breve podemos decir que el tema ha sido y es cuestionable en el campo de la enseñanza-aprendizaje de lenguas extranjeras. En la actualidad existe cierto consenso sobre el hecho de que el carácter nativo o no nativo del profesor no resulta relevante.Todavía, no ha podido demostrarse la superioridad del profesor nativo por el mero hecho de serlo (Moussu y Llurda 2008: 316). La oposición profesor nativo/no nativo no ha sido frecuentemente tratada en el campo del español como lengua extranjera pero resultan más abundantes los estudios sobre las creencias y las actitudes del profesorado y preferencias de los estudiantes sobre la condición del profesor.

En breve podemos decir que en la lingüística teórica, especialmente la chomskiana, el hablante nativo (como hablante-oyente ideal) es la única fuente fiable de datos lingüísticos (Chomsky 1965:48). Sin embargo, el 
carácter nativo/no nativo resulta difícilmente definible (Martínez Agudo, 2010:244-253).

Ahora bien, Si nos preguntamos cuál de los dos tipos de profesores puede resultar más exitoso en su labor docente, obtenemos dos respuestas, según Martín (1999: 433): el nativo puede desarrollar mejor la función de profesor por su conocimiento implícito de la lengua meta, sin embargo, el profesor no nativo tiene la experiencia de haber aprendido la lengua y está más capacitado para competir con los profesores nativos. Los estudios que existen actualmente constatan las diferencias entre ambos, sin llegar a establecer la superioridad de uno sobre otro (Martínez Agudo, 2010:254), pero sí descomponen alguna de las habilidades lingüísticas que tradicionalmente sonlas características de los profesores nativos, concretamente la capacidad extraordinaria para producir textos escritos fluidos y espontáneos y la habilidad de escribir creativamente. Lasdiferencias individuales justifican la exclusión de estos rasgos como características distintivas de todo hablante nativo. Medgyes, P. (1994:78), y entre otros autores (Martínez Agudo, 2010:244) presenta unas cuantas características positivas para el profesor no nativo y que podemos resumirlas en lo siguiente:

- Saber de cerca la cultura de los alumnos, su forma de pensar, sus hábitos de estudio, etc.

- Ofrece un buen modelo de aprendizaje.

- Enseña de manera efectiva estrategias de aprendizajepor haber estudiado la lengua meta.

- Proporciona más información sobre la lengua extranjera.

- puede conectar con más facilidad con las dificultades que experimentan losestudiantes.

- Tiene la ventaja de anticipar y predecir las posibles dificultades lingüísticas

- se adapta en muchas veces al ritmo de aprendizaje sus alumnos y les proporciona a lo largo del curso mucho ánimo y refuerzo positivo.

- El no nativo, aunque utilice la lengua extranjera en clase, siempre recurrirá a su lengua nativa para explicar ciertas dudas o matizar ciertas explicaciones gramaticales. 
Por tanto, según estos autores, las posibilidades de éxito profesional son similares para el profesor no nativo y para el nativo, siempre que el primero alcance la competencia comunicativa necesaria para enseñar la lengua y el segundo la formación necesaria. Otros estudios afirman que el profesor no nativo puede ser un modelo de éxito en el aprendizaje a través de facilitar el contraste entre la lengua y cultura de origen y de destino (Martínez Agudo, 2010:245). Por eso y como hemos señalado antes existe suficiente consenso sobre la necesidad de evitar la comparación entre los dos modelos y establecer una causalidad directa entre competencia comunicativa y prácticas pedagógicas. Las diferencias entre ambos tipos de profesores pueden resultar beneficiosas si contemplamos la posibilidad de colaboración entre ellos. Si el origen del profesor no es el factor que determina sus capacidades profesionales entonces ¿cuáles son los factores determinantes? Algunos educadores han determinado, además de la formación específica, otros factores. Algunos especialistas en la enseñanza del español como lenguas extranjera (Alfonso Hinojosa y Joaquín Martín ${ }^{3}$ ) consideran que un buen profesor de lenguas extranjeras no solo debe ser un usuario competente del lenguaje, con las habilidades necesarias para analizarlo, sino que además debe conocer una lengua extranjera a través de una experiencia formal de aprendizaje.

\section{2- La Metodología}

En la enseñanza de LE los métodos vigentes proporcionan ventajas diferentes entre sí. Hay métodos, por ejemplo, son partidarios de las tareas que se basan en la comunicación oral y otros en la comunicación no verbal tales como materiales visuales, y gráficos para dar explicaciones detalladasy acercarse másal contenido que se ha presentado verbalmente. En estos casos el profesor puede complementar el input lingüístico con más información. La dicotomía del input verbal - no verbal tiene sus frutos en la enseñanza de lenguas incluso a un nivel muy básico. En los niveles más avanzados, resulta más fácil comprender ciertas descripciones lingüísticas con la ayuda de input no verbal. Los nuevos aspectos de la LE tiene que ser presentados dentro de un contexto significativo antes de ser aprendidos por el alumno. Se debe integrar todo aquello que se deberá aprender en la lengua extranjera. Exponervarios textos que se basan más en la comunicación oral tiene sus ventajas sobre todo si la segunda lengua o lengua extranjera no se parece a 
la lengua materna (LM) del estudiante. El profesor debería ser capaz de poner en práctica múltiples actividades y enfoques, con el fin de que cada día sea un poco distinto. Con un poco de creatividad la clase puede ser variada e interesante $\mathbf{y}$ atraer y motivar más a los alumnos; a nadie le agrada dedicar horas y horas a mirar un libro de texto y a hacer ejercicios de rellenar huecos. Por lo tanto hay que pensar en otras salidas para fomentar la expresión oral con el fin de aumentar la competencia comunicativa en el aula para todos los estudiantes. La interacción oral es un recurso muy importante. Los ejercicios diseñados en los manuales tienen sus ventajas para este efecto y sobre todo los que constan normalmente de breves diálogos practicados sobre un modelo. Otra manera mucho más eficaz para activar la interacción oral es el uso de la lengua en la clase en varias situacione sobre todo con fórmulas repetitivas: como "Abre la puerta", "Cierra la ventana", preguntas "Cómo se dice en español ${ }^{4}$....etc. en un principio el alumno empieza a contestar a estas preguntas pero, con el tiempo se acostumbra a usar, a su vez, estas expresiones con normalidad ${ }^{5}$.En fin, estos métodos son ideales para explorar todo tipo de textos, desde una descripción hasta una argumentación, son eficaces para proporcionar unas herramientas excelentes para enseñar al alumno a actuar con libertad, opinar, defender posturas, expresar precepciones personales y evitar la memorización mecánica en el idioma extranjero.

Lo que hemos planteado puede servir como un punto de partida para acercar aún más a la situación creativa durante el aprendizaje del español como lengua extranjera. Pero está claro que, para los profesores de español como LE en Irak, el problema no es solamente cuestión de métodos; es un problema de convencer a los estudiantes a cambiar sus ideas y explicarles la importancia del aprendizaje de español y que ellos han elegido una lengua de gran importancia a nivel mundial y que perfeccionarla bien les puede a servir para crear un curriculumpráctico en el futuro. Una parte de esteproblema es eliminar las ideas estereotipadas negativas y remplazarlas porotras positivas $^{6}$.

Otro problema consiste en que los alumnos están acostumbrados acontestar a las preguntas propuestas por sus profesores pero no saben formularlas. A lo largo de la trayectoria de enseñanza del español como LE, los buenos e educados estudiantes, son aquellos que levantan sus manosuna vez que les 
haya preguntado para contestar en el momento apropiado, pero resulta muy extraño ver a un estudiante hacer una pregunta. Éste es como hemos dicho, el modelo vigenteen experiencia educativa de la mayoría de los profesores de lenguas extranjeras no solamente en Irak sino en otros países. Entonces, los profesores implicados en la enseñanza de LE, deberíamos cambiar esta perspectiva y dar a los alumnos la iniciativa de hacer preguntas. Hoy es tan evidente pero que durante muchos años no fue así.

A continuación presentamos algunas estrategias que, según las teorías modernas (Aguilera Terrats, 2007:58-60), han funcionado bien para entregar el "timón" a los estudiantes y cambiar el role, docente-estudiante, dentro de este ambiente. Así pues en la clase (sea de primer año, segundo, etc.), los estudiantes tienen que:

- preparar unas preguntas, hablando sobre, por ejemplo: materias que les interesan, sus propios objetivos; las relaciones entre sí; la situación de Irak después de 2003. cada estudiante a lo largo del curso, tiene que preparar esas preguntas con un compañero de clase antes de usarlas. El intercambio de preguntas ayuda a crear un ambiente interactivodentro de la clase y da confianza de poder usar la lengua ya. Éste puede ser el primer paso. Es muy interesante y lúdico cuando los estudiantes comienzan a hacer preguntas uno al otro sobre una noticia de mutuo interés o algo bastante intrincado. Los ejercicios de las preguntas es un ejemplo muy efectivo e interesante. En los ejercicios de preguntaslos alumnos pueden expresar sus observaciones sobre la vida cotidiana, el medio ambiente, etc., y los estudiantes y el profesor pueden aprender mucho de los intereses de la clase con esta idea. Enlas preguntas no es necesarioexigir respuestas, porque muchas pueden ser comentarios y observaciones de la vida cotidiana.

\section{Ejemplo $^{7}$ :}

- ¿Por qué vivimos?

- ¿Esto es lo que pasó cuando fuimos a Granada?

- ¿Merece la pena escucharlo otra vez?

- Leer los textos que comprenden varios modelos de entrevistas con personas destacables, tanto en el campo de la literatura como en otros campos de la vida, y también leer ejemplares de sus contribuciones. Los 
libros que presentan un buen modelo gramatical, con diálogos basados en situaciones auténticas pueden integrar algo de la cultura de la lengua meta(González Sánchez, 2016:15).

- Los estudiantes tienen que hacer entrevistas entre sí, y con otros que hayan estudiado en España, sea estudiante, o cualquier persona que haya tenido esta experiencia en aquel país. Otrométodo para resolver el problema de la integración de la gramática con la cultura en la enseñanza de la lengua a los alumnos en las primeras fases de aprendizaje. El método consiste en el usode las aplicaciones que tienen las nuevastecnologías en el aula. Según lo que explicó Martín (ver la nota $\mathrm{N}^{\circ} 3$ ), herramientas como el subtitulado, las aplicaciones gratuitas en las redes de internet, las pizarras digitales o la edición digital permiten introducir en la clase nuevos materiales, que facilitan mucho el trabajo de los profesores y les permiten conseguir una perfecta adaptación al idioma de los alumnos.

- Los estudiantes tienen que prepararuna lista de preguntas para una imprevista entrevista oral, esta tiene que ser grabada, y después analizada en la clase.

- Los estudiantes tienen que escribir composiciones sobre sus diarios para saber cuál es su nivel de español.

- Es imprescindibledejar los estudiantes en el aula solos sin la intervención del profesor, para crear una experiencia parecida a la llegada a un país extranjero. Es interesante ver cómo colaboran entre ellos cuando se dan cuenta de que están solos. Utilizar esta estrategia podría ser bueno para romper algunos estereotipos (por ejemplo, el castigo del profesor).

- Es importante notar que no son siempre los buenos estudiantes los que lo hacen bien. Muchas veces, hay estudiantes con menos nivel lingüístico pero más atrevidos.

- En la clase se puede usar una variedad de películas (incluso las de terror) para abrir discusiones sobre varios temas. Las películas son unos medios eficaces para una buena introducción a la cultura de la lengua meta, y a la vez ayudan mucho a los estudiantes a proporcionar temas sobre diversas situaciones.

- Los estudiantes tienen que escribir noticias actuales de interés, luego se comienza a analizarlos comentarios sobre las noticias que han escrito. 
Todas estas estrategias pedagógicas tienen como fin para dar un paso de acercamiento a la cultura de la LE, creando un deseo y una motivación en el estudiante. Tenemos que siempre recordar y reconocer que cada país es diferente de su manera de ser, cómo vivesu gente, su manera de pensar(intersección de la lengua con la cultura).

\section{3- Evaluación}

En la enseñanza tanto los manuales (la formación permanente del profesorado) como la lengua desempeñan un papel muy importante en la experiencia del aprendizaje. La normativa vigente y que actualmente en vigor en Irak para la enseñanza de las lenguas en general y el español en particular se basa de una manera claraen el marco de referencia de las academias mundiales de lenguas, la Real Academia Española de Lenguas y del Instituto de Cervantes correspondientemente. Y este hecho garantiza de una manera tajante, a pesar de lastendencias personales de cadaprofesor, un cierto consenso en las líneas básicas de la enseñanza.Pero hay que tener en cuenta que en la evaluaciónlos materiales deben tener prioridad sobre la corrección de la lengua. Algunos profesores hacen pruebas de evaluación que comprenden dos partes. La primera parte se basa en la destreza de comprensión en la cual se puede estructurar como prueba, escogiendo una respuesta entre varias posibles muestras. En este campo se valora sobre todo el conocimiento del alumno sobre el contenido de la asignatura. En segundo lugarse puede estar orientado más bien hacia el aspecto lingüístico con preguntas abiertas en las cuales los alumnos tienen que contestar en la lengua que están aprendiendo. De esta manera se pueden evaluar en la misma prueba tanto la competencia lingüística como la comunicativa. Hay que tener en cuenta,como hemos dicho anteriormente, quelos buenos manuales son aquellos que ofrecen muchas oportunidades de practicar oralmente la lengua meta, los que presentan numerosas actividades orales. Pero esto queda plantado e insuficiente sin el cambio en la actitud del profesor lo cual debe ser manifestado igualmente que en los manuales. 


\section{4- Conclusiones}

En nuestra propuesta hemos intentado ofrecerun acercamiento pedagógico,que no solamente sirve para superar algunos de los escollos de la enseñanza en Irak, sino también ofrecer un ejemplo bien específico con varias estrategias útiles y que no cuestan muchoesfuerzo, ni viajar ni gastar dinero para conseguirlas. Creemos que lo mejor en la enseñanza y el aprendizaje a la vez, es combinar en la cultura del otro lo práctico con lo idealista (intersección de la cultura con la lengua). Es muy práctico saber hablar más de una lengua, y con este poder podemos construir puentes o montar barreras. Ser un profesorno es una tarea tan fácil y serlo necesita una destreza. Los profesores no tienen que poseer solamente conocimientos y estar preparadostanto en didáctica como en metodología, sino hay que tener por dentro el placer de enseñar teniendo en cuenta lo siguiente:

- Ser abiertos a los cambios porque cada día salen a la vista métodos y técnicas nuevos.

- El contenido que va a enseñar y aprender debe ser útil y lúdico.

- Tener en cuenta las diferencias individuales de los alumnos partiendo de la idea de que cada estudiante tiene su propia manera de pensar.

- Los medios auditivos, visuales son muy importantes en el proceso de aprendizaje ya que estos ayudan a ofrecer situaciones cercanas a la realidad sobre todo en los países cuya cultura es distinta a la de la lengua meta.

- En el tema de la libertad y la autonomía del alumnado, los profesores tienen que servir como guías para resolver los problemas y dar los consejos.

- La relación entre profesor- alumno tiene que basarse en la confianza, el optimismo y el respeto, estos factores dan sus valiosos frutos en la creación de un ambiente agrado, sin miedo de preguntar, sentirse libre intimidados frente a una clase llena de alumnos al proponer las actividades.

- Por lo último, y creemos que es necesario establecer unos límites para el estudiantes para saber lo permitido y no lo es en el aula.

Finalmente podemos decir que las limitaciones de espacio nos impiden hablar de todo, pero esperamos que el acercamientopresentadoaquí sirva para que los que estamos involucrados en la enseñanza de lenguas 
extranjeras reflexionemos sobre él, reflexión de la que creemos que podrá contribuir a ampliar un poco la investigación sobre las dificultades que enfrentan los profesores no nativos sobre todo los del español como LE en Irak. Para que sean esos profesores percibidos por sus estudiantes, será necesario procurar en el futuro con unos estudios empíricos más profundos basados en cuestionarios o entrevistas que combinen la metodología cuantitativa con lacualitativa. Todo ello permitirá aumentar la validez de las conclusiones y ofrecer un panorama más concreto sobre las creencias de los estudiantes y su preferencia por profesores nativos o no nativos. De esta forma resultará más fácil eliminar lasideas estereotipadas la dicotomía de profesor nativo y no nativo. No hay que olvidar también que la educación no puede ser ajena al contexto en el que se desarrolla. Hoy, el desarrollo tecnológico y económico ha convertido a la globalización como una característica esencial que no se puede dejar fuera del proceso educativo.

\section{Bibliografía}

1. Aguilera Terrats, José Ricardo, Castillo Rosas, Adriana, García Sánchez. Percepción De Los Roles Docente-Estudiante: Problema que influye en la calidad de la enseñanza. Innovación Educativa [en linea] 2007, 7 (Mayo-Junio), consultada el día 21 de marzo de 2018.Disponibleen:http://sociales.redalyc.org/articulo.oa?id1794212 17004

2. Árva, V. y P. Medgyes (2000): "Natives and non-natives teachers in the classroom", System, 28 (3), 355-372.

3. Bloomfield, L. (1933): Language, New York: Henry Holt.

4. Braine, G. (1999). Los educadores no nativos en la enseñanza del inglés.Mahwah, NJ: Lawrence ErlbaumAssociates.

5. Castañeda Castro, A. (1994a). "Adquisición de la gramática de algunos aspectos del español: comprensión práctica". ActasDel II CongresoNacional de ASELE: 135. 142.

6. Castañeda Castro, A. (1994b). Procesos de Automatización y Prácticas Gramaticales Comunicativas (de la adquisición del subjuntivo en español como lengua extranjera). Tesis Doctoral. Universidad de Granada.

7. Chomsky, N. (1965). Aspects of the theory of syntax. Cambridge, MA: MIT Press.

8. González Sánchez M. (2016): Análisis metodológico de manuales de español para extranjeros: últimas aportaciones y perspectivas de futuro.

Disponible en(http://e- 
spacio.uned.es/fez/eserv/bibliuned:master-Filologia-FPESL-

Mgonzalez/Gonzalez_Sanchez_Maria_TFM.pdf consultada el día 10 de marzo de 2018).

9. Kerschensteiner G.(1949). ElAlma del Educador y el Problema de la Formación del Personal Docente. Múnich.

10. Luzuriaga L. 1957: 145. La institución libre de enseñanza y la educación en España. Universidad de Buenos Aires. Buenos Aires.

11. Madrid, D. (1998). Guía para la investigación en el aula de idiomas. Granada: Grupo Editorial Universitario.

12. Martín Martín, J. M., (1999): "El profesor nativo de español”, M. Franco et al. (eds.), Actas del X Congreso Internacional de ASELE. Nuevas Perspectivas en la Enseñanza del Español como Lengua Extranjera Cádiz: Servicio de Publicaciones de la Universidad de Cádiz. 1:

433-348 http://cvc.cervantes.es/ensenanza/biblioteca_ele/asele/asele_x.htm,co nsultada el día 7 de marzo de 2018.

13. Martínez Agudo, J. de D. La edad del profesor de lengua extranjera y su condición de nativo-no nativo: creencias del profesorado en formación. Revista de Lenguas Modernas, $N^{\circ}$ 13, 2010 / 241-257 / ISSN: 1659-1933.

14. Matsuda, A. y Kahmi-Stein. (2004): "Autonomy and collaboration in teacher education", Journal sharing among native and nonnative English-speaking teachers, Kamhi-Stein (ed.), Learning and teaching from experience: Perspectives on nonnative English-speaking professionals, Ann Arbor: University of Michigan Press.: 176-189.

15. Medgyes, P. (1994): The Non-Native Teacher. Londres: MacMillan.

16. Montero Mesa, M. L. (2000). La formación del profesorado ante los retos de la multiculturalidad.Revista de currículum y formación del profesorado, 4 (1).

17. Moussu, L. y E. Llurda (2008): "Non-native English-speaking English language teachers: History and research", Language Teaching, Cambridge UniversityPress.DisponibleEnhttp://www2.warwick.ac.uk/fac/soc/al/ research/groups/ellta/elted/events/download.pdf (Consultada el día 10 de mayo de 2018).

18. Rubio Gel, A y Álvarez Irarreta, A. (2010). Formación de Formadores después de Bolonia. Madrid, Díaz de Santos, S. A.

19. Santos Gargallo, I. (1993): Análisis Contrastivo, Análisis de Errores e Interlengua en el marco de la Linguística Contrastiva. Madrid, Síntesis. 
${ }^{1}$ Segunda lengua y lengua extranjera son dos términos que no se refieren a la misma cosa. Segunda lengua (L2): aquella lengua que cumple una función social e institucional en la comunidad lingüística en que se aprende, es decir es la lengua que se adquiere en un contexto natural de forma parecida a cómo se ha adquirido la lengua materna en muchos aspectos, tenemos por ejemplo, el francés, que se considera la lengua segunda en Túnez y Argelia donde el árabe es la lengua materna. Lengua extranjera (LE): aquella lengua que se aprende en un contexto en el que carece de función social e institucional; el español es lengua extranjera en estos dos países.

${ }^{2}$ Se piensa que la edad del profesor puede influir en la metodología de enseñanza desarrollada en el aula, aunque no existe ningún dato concreto concluyente al respecto que nos permita confirmar si se aprende más o enseñan mejor los profesores jóvenes frente a los mayores o si más bien sucede lo contrario. Pero el tema de la edad y su apreciación fue planteado detalladamente por muchos autores (véase Martínez Agudo, 2010: 243).

${ }^{3}$ Innovación metodológica en la enseñanza de idiomas, Disponible en http://noticias.universia.es/vida-

universitaria/noticia/2012/08/14/958192/innovacion metodologica-ensenanzaidiomas.html consultada el día 14 de mayo de 2018.

${ }^{4}$ Todos los ejemplos citados en este trabajo ha sido ideados por nosotros.

${ }^{5}$ Con la meta que ha conseguido la corriente del enfoque comunicativo en la enseñanza de lenguas, la teoría cognitiva ha mostrado que "hablar" es una destreza que exige adiestramiento y que la automatización de las estructuras sintácticas parte integradora de la comunicación- reclama ejercicios variados, graduales y continuados. Así pues, la influencia de la instrucción en la ASL no se determina sólo por prestar atención a la relación forma /función, sino también por el efecto de la práctica repetitiva, ya que, según Ellis, la práctica controlada persigue la automatización del conocimiento declarativo que ya posee el aprendiz, de modo que debe considerase como una fuente de input (input que sirve para que desarrollemos nuestro conocimiento lingüístico). «La posesión de procesos automatizados que liberen capacidad de procesamiento controlado puede lograrse mediante la conversión, con la práctica repetida, de procesos controlados en procesos automáticos». (Castañeda, 1994b:115).

${ }^{6}$ En Irak existe solamente un departamento de español en la Facultad de lenguas en la Universidad de Bagdad donde se otorga el título de Licenciado en Españoltambién se da el español como asignatura optativa o troncal dentro de la licenciatura de otros idiomas en algunos departamentos tanto en la misma facultad como en otras facultades (por ejemplo en Facultad de Filosofía y Letras/ Universidad de Bagdad). En la enseñanza secundaria, el español se imparte en sólo algunos colegios de secundaria en Bagdad como asignatura troncal.

${ }^{7}$ Ver la nota $\mathrm{n}^{\circ}$ 
الصعوبات والمشاكل في تعليم و تعلم اللغة الاسبانية بشكل خاص واللغات الاجنبية بشكل عام: الكادر التدريسي الاجنبي

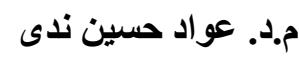
وزارة التعليم العالي و البحث العلمي / دائرة البعثات و العلاقات الثقافية خلاصة البحث

يهدف هذا البحث إلى تزويد أعضاء الهيأة التدريسية التدريسي بالمهارات اللازمة ليكونوا قادرين على التفاعل في مع الحالات المختلفة والتعامل مع المشاكل اليومية التي تواجه الطلبة متعلمي اللغة الاسبانية بوصفها لغةً ثانية. ان بحثنا هذا هو محاولة لتمكين اساتذة اللغات الاجنبية على

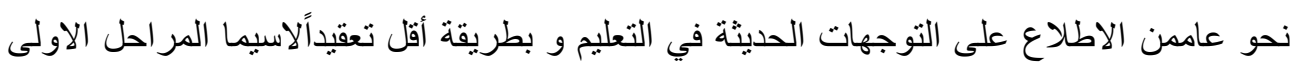
في تعلم اللغة الاجنبية. الكلمات المفتاحية: تعليم, اللغة الاجنبية, الاسبانية, الهيأة التدريسية, طرق تدريس.

\section{About the author}

Por Inst. Awad Hussein Nada (Ph.D.)

Ministerio de Educación Superior e

Investigación Científica- Dirección de

Becas y Relaciones Culturales

- Mail: awadhussein2002@yahoo.es

- $\quad$ M.N. 07813361057 